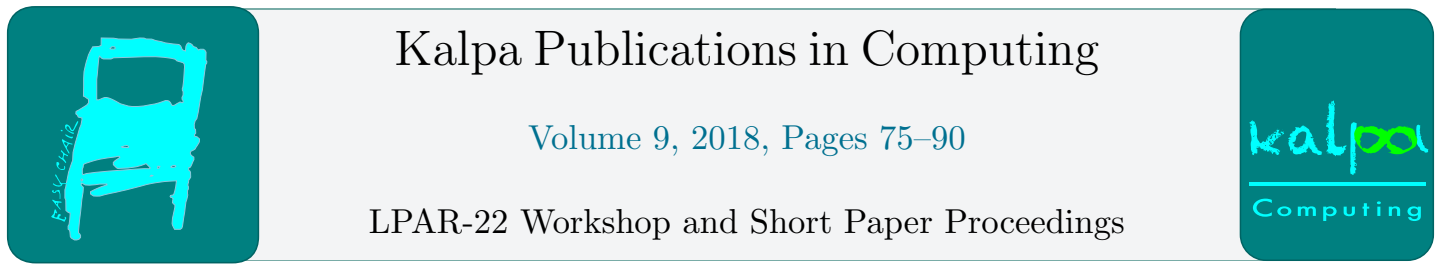

\title{
HoTT-Crypt : A Study in Homotopy Type Theory based on Cryptography
}

\author{
Paventhan Vivekanandan \\ Indiana University, Bloomington, Indiana, USA \\ pvivekan@umail.iu.edu
}

\begin{abstract}
This paper investigates a preliminary application of homotopy type theory in cryptography. It discusses specifying a cryptographic protocol using homotopy type theory which adds the notion of higher inductive type and univalence to Martin-Löf's intensional type theory. A higher inductive type specification can act as a front-end mapped to a concrete cryptographic implementation in the universe. By having a higher inductive type front-end, we can encode domain-specific laws of the cryptographic implementation as higher-dimensional paths. The higher inductive type gives us a graphical computational model and can be used to extract functions from underlying concrete implementation. Using this model we can extend types to act as formal certificates guaranteeing on correctness properties of a cryptographic implementation.
\end{abstract}

\section{Introduction}

Formal methods are used to verify that a system behaves in an expected way based on its specification. Type system, a lightweight formal method, is a tool for reasoning about programs which can categorically prove the absence of some bad program behaviors. In the early days of programming, type systems were used to ensure certain basic correctness properties of programs such as the arguments to primitive arithmetic operations are always numbers and differentiating between a string and integer value in the memory. During the twentieth century, types have become standard tools in logic, particularly in proof theory. One of the significant work in this area is a predicative modification of Church's type system proposed by Per Martin-Löf now known as Martin-Löf type theory [25][24]. It gives a computational interpretation to intuitionistic higher-order logic based on Russell's theory of types [23]. This extended type systems from tools merely ensuring correctness properties into first-class logics.

Homotopy type theory [1] extends Martin-Löf intensional type theory by adding higher inductive type and univalence axiom. In homotopy type theory, the witness or proof element of a type can be viewed as a point in a topological space, and a witness of an identity type can be viewed as a path in a topological space. A higher inductive type differs from an ordinary inductive type by providing constructors not only for points but also for paths. In particular, higher inductive types provide a natural encoding of many otherwise-difficult mathematical concepts, and univalence lets us work in our type theory the way we do on paper: up to 
isomorphism. Homotopy type theory, however, is not yet done. We do not yet have a mature theory or a mature implementation. While work proceeds on prototype implementations of higher-dimensional type theories [26][27], much work remains before they will be as convenient for experimentation with new ideas as Coq, Agda, or Idris is today. In the meantime, it is useful to be able to experiment with ideas from higher-dimensional type theory in our existing systems.

Homotopy type theory has thus far primarily been applied to the encoding of mathematics, rather than to programming. Nevertheless, some preliminary applications of homotopy type theory in programming have been investigated. For example, the work of [8] apply ideas related to homotopy type theory to modeling variable binding. Containers [28][29] in homotopy type theory can be used to implement data structures such as multisets and cycles. Patch theory [2] shows modeling of Darcs [32] version control system using the concepts of homotopy type theory.

Application of logic to novel problems raises numerous interesting research issues which could drive the progress in the theory. In this paper, we investigate a preliminary application of homotopy type theory in cryptography and discuss its practical limitations from an application perspective. More specifically we discuss how to specify a cryptographic scheme, which is deterministic, using the features of homotopy type theory. Formal verification of cryptographic protocols has become a significant research focus over recent years [5] [14]. Some widely used cryptographic implementations were found to be flawed after their deployment becoming vulnerable to various attacks. For example, the Heartbleed attack (CVE- 2014-0160) is a consequence of a simple coding error [13]. Even with skilled designers, developers and testers it is highly difficult to implement a cryptographic protocol without errors [12].

We discuss specifying the correctness properties of a cryptographic implementation using higher inductive types, implemented in Agda, and how to project computational models from such specifications. A cryptographic system which expresses decryption as an inverse of encryption [4] can be defined using a higher inductive type representing a graphical model in a topological space. A concrete implementation of the cryptographic system can be projected from this graphical model using univalence axiom. The higher inductive type acts as an abstract model for the encoded cryptographic system and enables us to specify the correctness properties as paths or higher-dimensional paths in a topological space. We investigate the practical application of homotopy type theory to an industry level cryptographic protocol, the cryptDB which employs multiple encryptions. We discuss the conceptual and the implementation concerns and analyze the challenges from an application perspective discussing the limitations and the future work. In short, we show how to extend types to act as formal certificates guaranteeing on various correctness properties of a cryptographic scheme. Mainly we make the following contributions.

- We show how to design a cryptographic construction using a higher inductive type and how to map the abstract type to a concrete implementation in the universe. Such developments give rise to interesting homotopies which are paths between paths or two-dimensional paths in a topological space.

- Paths in a higher inductive type are used to model correctness rules such as functional correctness [4], which says decryption inverses encryption, and this structure will be preserved in the mapping to the universe due to the functoriality of mappings in homotopy type theory.

- We can enforce various restrictions on the concrete implementation when a cryptographic 
protocol is modeled using a higher inductive type. We discuss designing a higher inductive type for a database model with multi-layered encryptions in the style of cryptDB [3].

- We discuss encoding of domain-specific properties related to homomorphic encryption, deterministic encryption, and order-preserving encryption as path between paths or homotopies in a higher inductive type.

We use the singleton framework [2] to implement the cryptDB protocol model discussed in this paper. This framework can be generalized to support the class of protocols implemented with encryption schemes that can be expressed using a contractible type. This limitation is imposed by homotopy type theory which requires the paths to be bijective. However, we do not have a better framework yet to implement non-bijective constructions. Designing cryptographic constructions as a higher inductive type has the following benefits.

- In type theory all functions are functorial. Therefore, the functional correctness and domain-specific properties of a cryptographic construction can be specified as paths or homotopies in a higher inductive type, and the functions will preserve the path structures in the mapping of the type to the universe.

- By specifying cryptographic properties as paths, we achieve guarantee on the correctness of the underlying concrete implementation with respect to the encoded properties.

- We can have a graphical representation of a cryptographic construction in a topological space, and we map it to a concrete implementation in the universe.

- By modeling a cryptographic construction as a higher inductive type, we can get the groupoid structure and the relevant coherence laws related to the higher inductive type for free.

- We will get a non-dependent eliminator also known as the recursion principle, and we can use it to define functions or to map the elements including the paths of the higher inductive type to elements of other types such as the universe.

- We will get a dependent eliminator also known as induction principle which can be used to formulate and prove theorems related to a cryptographic construction encoded as higher inductive type.

In the next section, we will discuss the different components of homotopy type theory. In section 3 we will give an example of encoding a simple cryptographic scheme, the one-time pad, using higher inductive type and explain how to map this higher inductive type to a concrete implementation of the scheme in the universe. In section 4, we will discuss how to design higher dimensional paths to enforce restrictions on the implementation of an industry level cryptographic protocol, the cryptDB. In section 5, we will review the implementation details, and in section 6 we will discuss the related work. In section 7 , we will see the limitations and future work before concluding.

\section{Background}

A formal specification of a cryptographic scheme requires a programming language with support for theorem proving. Proof-assistants with a strong mathematical background such as Agda and Coq can be used to specify correctness and security properties of a cryptographic construction. 
There are works which use an embedded domain-specific language [5] [14] [18] on existing theorem provers to support defining and proving cryptographic properties. In this paper, we discuss a new approach to specify cryptographic protocols based on types. This approach involves correlating a type with a cryptographic implementation. By combining with the right type, we can guarantee on various correctness properties of the cryptographic application. In the remainder of this section, we discuss the tools of homotopy type theory which are instrumental in modeling and associating types with cryptographic implementations.

Unlike set theory, which is an interplay between propositions and sets, type theory is based on the interpretation of propositions-as-types. According to this interpretation, a proposition stating that two elements of a type $a, b: A$ are equal corresponds to a type known as the identity type given by $a={ }_{A} b$ or $I d_{A}(a, b)$. In homotopy type theory, elements of the identity type $a={ }_{A} b$ are used to model the notion of paths or equivalences between $a$ and $b$ in the space $A$. An element of the type $a={ }_{A} b$ is a witness or a proof stating that $a$ and $b$ are propositionally equal. Propositional equality is a proof relevant notion of equality expressed by identity types. There is also a proof-irrelevant notion of equality in type theory known as judgmental equality or definitional equality. Definitional equality is not internal to the theory, and it is used to express equality by definition. For example, when we have a function $f: N a t \rightarrow N a t$ defined as $f(x)=x^{3}$ then $f(2)$ is definitionally equal to $2^{3}$.

Homotopy type theory extends Martin-Löf's intensional type theory by adding univalence axiom and higher inductive types. It introduces the notion of viewing type as a topological space in homotopy theory or a higher-dimensional groupoid in category theory. Because of this correspondence, we can observe an element of the identity type $x={ }_{A} y$ for $a, b: A$ as a path in a topological space or a morphism in a groupoid. Also, an element of the iterated identity types $m={ }_{x={ }_{A} y} n$ and $p==_{m={ }_{x=A} y} q$ can be viewed as a 2-dimensional and a 3-dimensional path respectively in a topological space or a morphism between morphism and a higher-level morphism respectively in a groupoid and so on.

A morphism at a level $k$ in a groupoid is called a $k$-morphism. A $k$-morphism has a groupoid structure defined by identity, composition, and inverse operations. These operations satisfy the groupoid laws which are associativity of composition, identity as a unit of composition and cancellation of inverses through a weak sense of equality but only up to a morphism at the next level $k+1$. We can view the $k$-morphism as a $k$-dimensional path in a topological space. Similarly, we can observe the elements of an iterated identity type at level $k$ as $k$-dimensional paths. Therefore a proof element of the type $x=_{A} y$ acts like a one-dimensional path between endpoints $x$ and $y$ and a proof element of type $m={ }_{x={ }_{A} y} n$ acts like a 2-dimensional path or a homotopy between paths of type $x={ }_{A} y$ and so on. Moreover, these paths also satisfy the groupoid laws up to homotopy at the next level in the following sense.

- $r e f l \circ x=x \circ r e f l=x \longrightarrow$ identity as a unit of composition

- $(x \circ y) \circ z=x \circ(y \circ z) \longrightarrow$ associativity of composition

- $! x \circ x=x \circ ! x=r e f l \longrightarrow$ cancellation of inverses

where $r e f l$ is an element of type $x={ }_{A} x$

Because of the correspondence of types to a topological space or a higher-dimensional groupoid, we can map the elements of an identity type, which are paths in homotopy type theory, to equivalences between types in a universe. Equivalence can be relaxed to a bijection when types behave like sets. The mapping of a path to equivalence is made possible by the univalence axiom which describes that we may identify equivalent types $A$ and $B$ in the following 
sense.

$$
u a:(A \simeq B) \rightarrow\left(A==_{U} B\right)
$$

In (1), the type $U$ is the universe or the type of types. The univalence axiom states that when we have a proof of type $A \simeq B$, we can obtain a path between $A$ and $B$. In homotopy type theory, the following defining equations give an equivalence between type $A$ and type $B$.

$$
\begin{gathered}
A \simeq B: \equiv \sum_{f: A \rightarrow B} i \operatorname{sequiv}(f) \\
i \operatorname{sequiv}(f): \equiv\left(\sum_{g: B \rightarrow A}\left(f \circ g \sim i d_{B}\right)\right) \times\left(\sum_{h: B \rightarrow A}\left(h \circ f \sim i d_{A}\right)\right)
\end{gathered}
$$

A homotopy between non-dependent functions $f_{1}, f_{2}: A_{1} \rightarrow A_{2}$ is given by the following equation.

$$
f_{1} \sim f_{2}: \equiv \prod_{x: A_{1}}\left(f_{1}(x)={ }_{A_{2}} f_{2}(x)\right)
$$

In (3), the composite $f \circ g$ is homotopic to the identity function $i d_{B}$, and the composite $h \circ f$ is homotopic to the identity function $i d_{A}$. There is also a reduced notion of equivalence called quasi-inverse. A quasi-inverse for a function $f: A \rightarrow B$ is given by

$$
q i n v(f): \equiv \sum_{g: B \rightarrow A}\left(\left(f \circ g \sim i d_{B}\right) \times\left(g \circ f \sim i d_{A}\right)\right)
$$

Also, we have a function that maps an element of quasi-inverse qinv $(f)$ to isequiv $(f)$ for $f: A \rightarrow B[1]$.

$$
\text { mkqinv }: \operatorname{qinv}(f) \rightarrow \operatorname{isequiv}(f)
$$

For examples described in this paper, we will use mkqinv to obtain a proof of equivalence from quasi-inverse. For a path $p: A=_{U} B$, we have a function coe [2] that coerces along $p$. The following equation gives the type of coe.

$$
\text { coe }:\left(A={ }_{U} B\right) \rightarrow(A \rightarrow B)
$$

In the presence of univalence, we also have a computation rule for coe [2] defined as follows.

$$
\text { coe }(\text { ua }(f, \operatorname{isequiv}(f))) x=f(x)
$$

where $x: A, f: A \rightarrow B$ and $(f, \operatorname{isequiv}(f)): A \simeq B$.

Higher inductive types are a general schema for defining new types in homotopy type theory. It extends an ordinary inductive type by providing constructors for generating paths and higher paths. In homotopy type theory, we define a higher inductive type by specifying its introduction, elimination, and computation rules. The introduction rule of a type specifies its constructors. The elimination rule of a type defines how to use its elements, and the computation rule describes the action of the elimination rule on the constructors of the type. A simple example for higher inductive type is the interval type $I$. It consists of two point constructors $0_{I}$ and $1_{I}$ and a path constructor seg : $0_{I}={ }_{I} 1_{I}$. The following declaration ${ }^{1}$ specifies the introduction rule for $I$.

\footnotetext{
${ }^{1}$ In this paper, we have given a reduced declaration of higher inductive types for better understanding. In Agda, we use rewrite rules to define higher inductive types.
} 


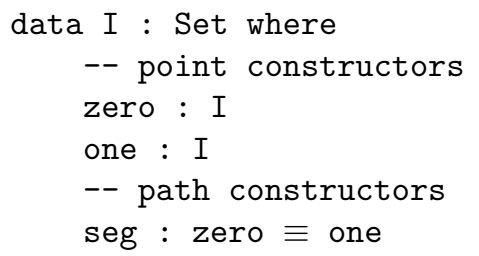

The non-dependent elimination rule or the recursion principle of I states that when given a type $C$ along with constructors $c_{0}, c_{1}: C$ and cseg $: c_{0}={ }_{C} c_{1}$, there is a function $f: \mathrm{I} \rightarrow C$ such that $f($ zero $)=c_{0}, f$ (one) $=c_{1}$ and $a p_{f}($ seg $) \equiv$ cseg where $a p_{f}$ defines the action of functions on paths. The equalities $f($ zero $)=c_{0}, f($ one $)=c_{1}$ and $a p_{f}(\mathrm{seg}) \equiv$ cseg are the computation rules for the type I. The computational rules for the point constructors zero and one hold definitionally, but the computation rule for path constructor seg holds only propositionally, and we specify it as an axiom which is a limitation of homotopy type theory.

Similarly, the dependent eliminator or the induction principle of I states that when given a type $D: I \rightarrow U$ along with constructors $d_{0}: D$ (zero), $d_{1}: D$ (one) and $d s e g: d_{0}={ }_{\text {seg }}^{D} d_{1}$, there is a dependent function $f: \prod_{(x: I)} D(x)$ with computation rules $f$ (zero) $=d_{0}, f$ (one) $=d_{1}$ and $a p d_{f}(\mathbf{s e g}) \equiv d s e g$. Here $d s e g$ is a heterogeneous path transported over seg and $a p d_{f}$ defines the action of functions on heterogenous paths [1].

Another important concept of homotopy type theory which is central to understand the idea proposed in this paper is that the functions behave functorially on paths. It means that a function $f: A \rightarrow B$ respects equality and it preserves the path structure in the mapping from type $A$ to type $B$. Now we can give the type of $a p_{f}$ which defines the action of non-dependent functions on paths as follows.

$$
a p_{f}:\left(x={ }_{A} y\right) \rightarrow\left(f(x)={ }_{A} f(y)\right)
$$

The following equation gives the action of dependent functions of type $f: \prod_{(x: A)} B(x)$ on paths.

$$
\operatorname{apd}_{f}: \prod_{p: x=y}\left(p_{*}(f(x))={ }_{B(y)} f(y)\right)
$$

In (10), $p_{*}(f(x))$ lying in space $B(y)$ can be thought of as an endpoint of a path obtained by lifting $\mathrm{p}$ from $\mathrm{f}(\mathrm{x})$ to a path in the total space $\sum_{(x: A)} B(x) \rightarrow A$ [1]. The following equation gives the type of $p *$ also known as transport.

$$
\text { transport }_{p}^{B}: B(x) \rightarrow B(y)
$$

where $p: x=y$ for $x, y: A$.

\section{Higher Inductive Type front-end for OTP}

In this section, we will discuss an encoding of the one-time pad using a higher inductive type with a path constructor to specify the encryption function. We will construct a proof for an equivalence which reflects the encryption path of the higher inductive type in the universe. The functional correctness property, which states that decryption inverts encryption, will be part of the construction of the proof for the equivalence. We will then map this higher inductive type, with the encryption path, to a concrete implementation of the one-time pad, with the equivalence reflecting the encryption path, in the universe. The encryption and the decryption functions are then projected from the concrete implementation in the universe using the higher 
inductive type which acts as a front-end. By accessing the concrete implementation of the one-time pad through a higher inductive type, we can get a certificate or a guarantee on the functional correctness of the system. Some other property such as homomorphic encryption requires introducing higher-dimensional paths to act as a certificate. We will discuss higherdimensional paths in section 4.

\subsection{One-time Pad}

The following Agda code gives the higher inductive type encoding of the one-time pad.

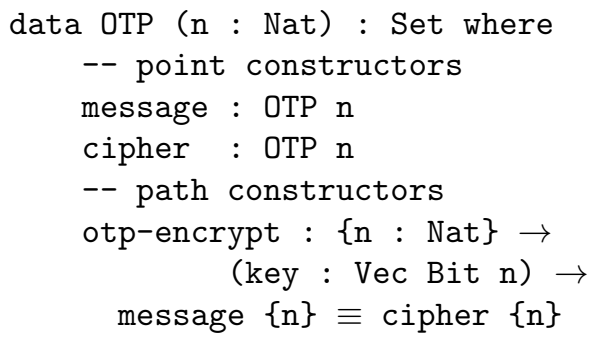

The higher inductive type OTP has two point constructors message and cipher representing the plain-text and the cipher-text respectively. The path constructor otp-encrypt represents the encryption function of the one-time pad. We parameterize the type OTP with the length $n$ of the data. otp-encrypt uses the same length parameter $n$ to specify the length of the key which encodes another restriction, namely the length of the key for the one-time pad should be equal to the length of the message, which is crucial for the security of the one-time pad.

The following code gives the recursion principle and its action on constructors or the computation rules for the type OTP.

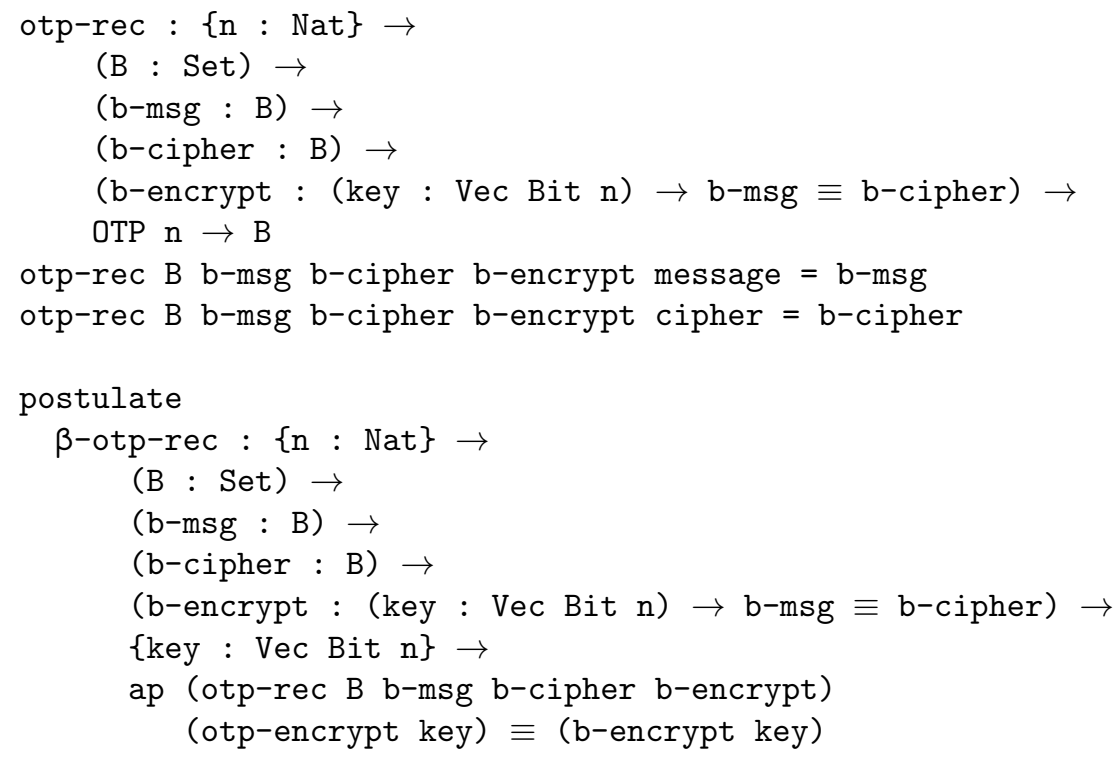

The recursion principle otp-rec states that when given a type B with point constructors $\mathrm{b}$-msg and b-cipher and path constructor b-encrypt, there exists a function of type OTP $\mathrm{n} \rightarrow$ B. otp-rec maps message and cipher to b-msg and b-cipher respectively. $\beta$-otp-rec gives 
the action of otp-rec on the path (otp-encrypt key) which maps it to the path (b-encrypt key). Equation (9) gives the type of ap. The computation rules for point constructors message and cipher are given as definitional equalities specified as part of otp-rec. The computation rule for the path otp-encrypt is postulated as propositional equality.

The following code gives the induction principle and its computation rules for OTP.

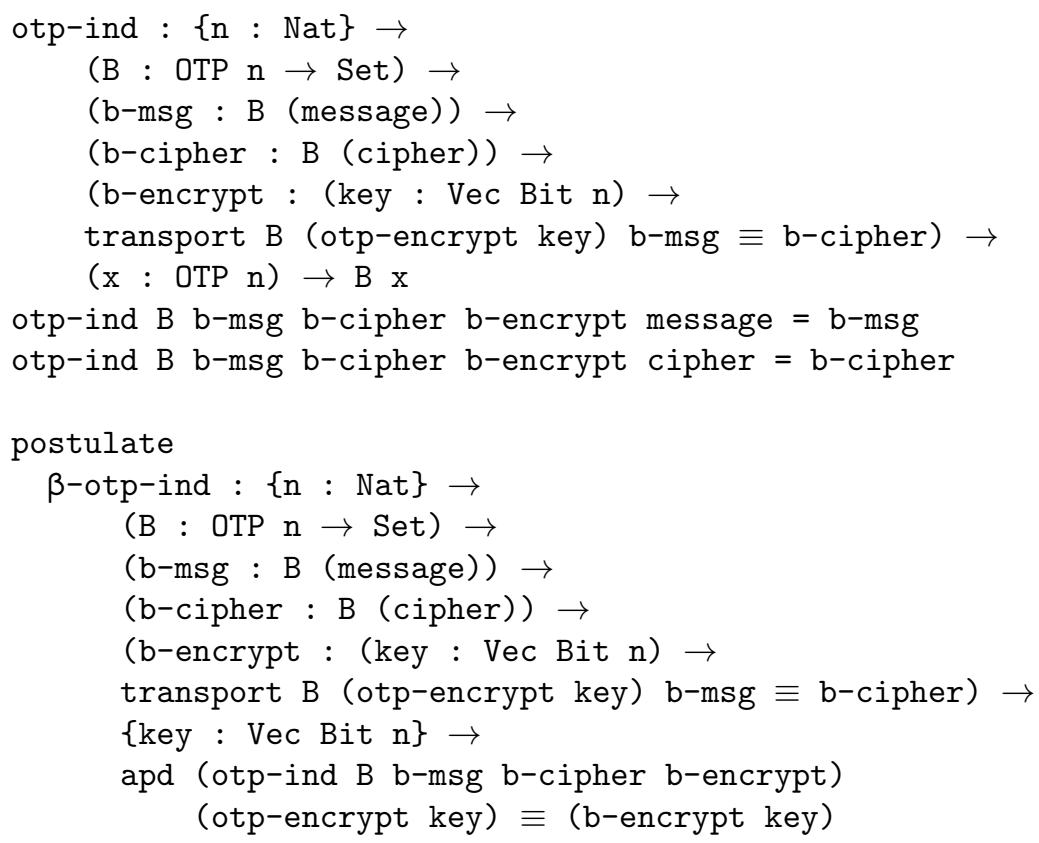

The induction rule otp-ind states that when given a type B : OTP $\mathrm{n} \rightarrow$ Set along with points $b$-msg, b-cipher and path b-encrypt, there exists a dependent function ( $x:$ OTP $n$ ) $\rightarrow$ B $\mathrm{x}$. The computation rule for path b-encrypt is postulated as propositional equality. Equation (10) gives the type of apd and equation (11) gives the type of transport where $p$ is the path (otp-encrypt key).

\subsection{Implementation of one-time pad in the universe}

The functional programming aspect of homotopy type theory allows us to implement any cryptographic schemes. In this section, we will develop a concrete model for the higher inductive type OTP described in section 3.1. The encryption function for the one-time pad is straightforward, and it is implemented using xor. The encryption of one-time pad is defined using the following function.

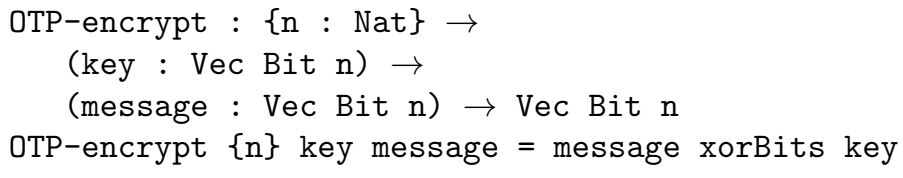

where xorBits perform xor on two vectors of equal length.

Similar to keys, we have chosen to use the type Vec Bit $\mathrm{n}$ to represent the point constructors message and cipher of the higher inductive type OTP in the universe. Therefore, the path 
otp-encrypt should be mapped to an equivalence formed by OTP-encrypt between types Vec Bit $\mathrm{n}$ and Vec Bit $\mathrm{n}$. To create an equivalence for the function OTP-encrypt, we need a proof element of type given by equation (5). To construct a proof element of (5), we need a function $\mathrm{g}:$ Vec Bit $\mathrm{n} \rightarrow$ Vec Bit $\mathrm{n}$, a proof element of $\mathrm{f} \circ \mathrm{g} \sim \mathrm{id}$, and a proof element of gof $\sim$ $i d$. For the one-time pad, the encryption function is also its inverse. So both $f$ and $g$ are represented by OTP-encrypt in this case. Therefore, the types fog $\sim$ id and gof $\sim$ id are definitionally the same. The equivalence formed by OTP-encrypt is defined as follows.

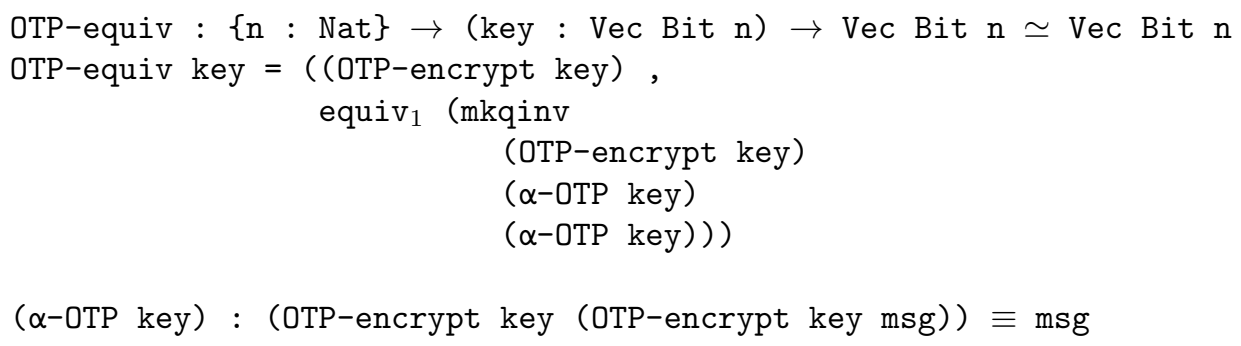

In the above code, (OTP-equiv key) is of the type given by equation (2). equiv forms a proof element of the type given by equation (3). The type of mkqinv is given by equation (6) which takes an element of (5) as input and gives an element of (3) as output. ( $\alpha$-OTP key) is a proof which says the encryption of msg, implemented by OTP-encrypt, followed by its decryption, which is also implemented by OTP-encrypt in this case, is the same as msg.

\subsection{Mapping OTP into the universe}

The higher inductive type OTP defined in section 3.1 can now be mapped into the universe using univalence. The abstract nature of higher inductive types also means that we can map the same type to more than one concrete implementation in the universe whenever compatible. The equivalence (OTP-equiv key) respects the path structure specified by the constructor otp-encrypt. Because of this, a path formed by univalence given by (ua (OTP-equiv key)) represents the path structure of otp-encrypt in the universe. This correspondence allows us to define a mapping I-OTP which maps the points message, cipher of OTP to type Vec Bit $\mathrm{n}$ and a mapping I-OTP-path which maps the path (otp-encrypt key) to (ua (OTP-equiv key)).

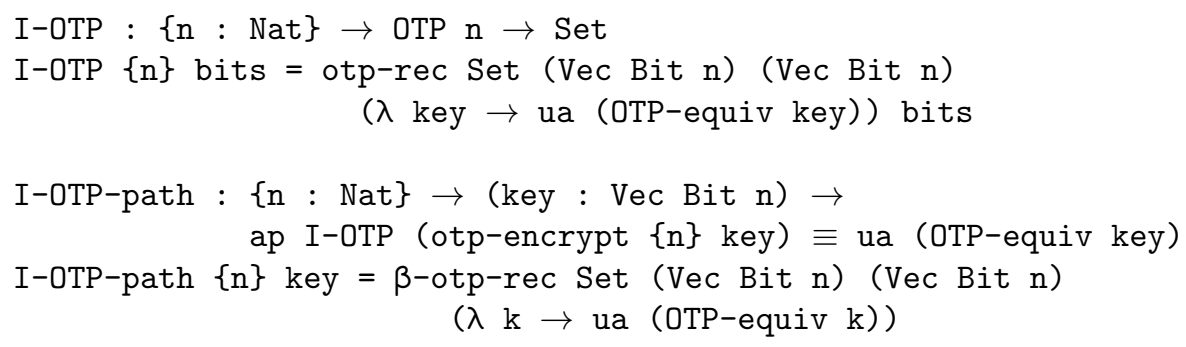

I-OTP is defined using the recursion principle otp-rec of the higher inductive type OTP. It maps the points of OTP to the type Vec Bit $\mathrm{n}$ in the universe represented by Set. I-OTP-path maps the path (otp-encrypt key) to (ua (OTP-equiv key)) using $\beta$-otp-rec. Now we can define an interpreter function ITP using coe given by equation (7) as follows.

ITP $:\{\mathrm{n}:$ Nat $\} \rightarrow\{\mathrm{a}$ b : OTP $\mathrm{n}\} \rightarrow$

$(\mathrm{p}: \mathrm{a} \equiv \mathrm{b}) \rightarrow$ 


$$
(\mathrm{I}-\mathrm{OTP} \text { a) } \rightarrow \text { (I-OTP b) }
$$

$\operatorname{ITP}\{n\}\{a\}\{b\} p=$ coe (ap I-OTP $p$ )

When we give the path otp-encrypt as input, the interpreter ITP returns the encryption function OTP-encrypt. By accessing a concrete implementation in the universe using a higher inductive type, we get the certificate or guarantee specified by the path structures of the higher inductive type. In the case of OTP, the functional correctness property is part of the equivalence (OTP-equiv key) given by ( $\alpha$-OTP key), and the path otp-encrypt will reflect this through the mapping specified by I-OTP-path.

We will consider an example of using ITP to extract OTP-encrypt and its application on a vector.

pf : (ITP (otp-encrypt (1b::(0b::[]))) (1b::(1b::[])))

$$
\equiv(\mathrm{Ob}::(1 \mathrm{~b}::[]))
$$

In the above code, ITP takes otp-encrypt as input with key (1b :: (Ob :: [] $)$ ) and plain-text (1b :: (1b : : [] $))$ and returns the cipher-text (0b :: (1b ::[])) as output.

\section{Encoding Properties as Higher Dimensional Paths}

The path otp-encrypt described in the previous section is one-dimensional. We can also encode domain-specific cryptographic properties as higher dimensional paths. In this section, we will design properties of a database model with multi-layered encryptions in the style of cryptDB [3] as higher dimensional paths. CryptDB has different layers of encryption known as onion layers of encryption. The idea of cryptDB is to allow computation on top of encrypted data without the need to decrypt them. For example, homomorphic encryption can be used to implement addition, and deterministic encryption can be used to perform equality comparison on top of encrypted data. Similarly, order-preserving encryption can be used to implement inequality comparisons on encrypted data. A higher inductive type can be used to define the computational behavior of cryptDB. We will consider the following higher inductive type specification to discuss encoding domain-specific laws of cryptDB as higher dimensional paths ${ }^{2}$. CryptDB involves non-bijective functions, and can be implemented using singleton types [2]. In this section, we will not be focusing on the implementation details or mapping types into the universe.

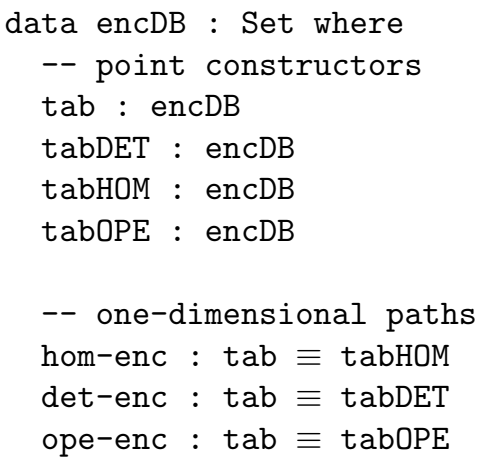

\footnotetext{
${ }^{2}$ We have simplified the higher inductive type encDB for ease of understanding. Please see section 5 for implementation details.
} 


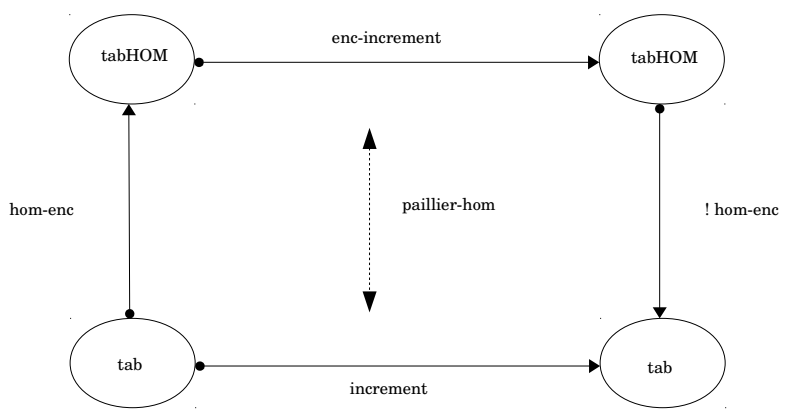

Figure 1: Homotopy representing the homomorphic property of paillier cryptosystem. The path hom-enc concatenated with enc-increment and (! hom-enc) is equal to the path increment.

The higher inductive type encDB specifies a lot of restrictions and a mapping to a concrete implementation should respect those restrictions. For example, it says that homomorphic encryption is a function that takes a plain-text table tab as input and gives an encrypted version of the table tabHOM as output. The inverse path (! hom-enc) specifies the decryption function. Similarly, the paths det-enc and ope-enc specifies the deterministic and order-preserving encryption schemes respectively. The higher inductive type encDB acts as a single interface giving a lot of information on underlying implementation of a cryptographic setting. It provides us with a graphical model composed of points, paths, paths between paths or higher dimensional paths to specify the correctness properties and various domain-specific laws of a cryptographic construction. In the remainder of this section, we will discuss homotopies or path between paths describing properties specific to homomorphic encryption, deterministic encryption, and order-preserving encryption.

\subsection{Homomorphic Encryption}

Homomorphic encryption can be used to perform computations on cipher-text. In cryptDB, homomorphic encryption is implemented using paillier cryptosystem. According to the homomorphic property of paillier cryptosystem [16], the addition of two plain-texts will be equal to the multiplication of their corresponding cipher-text. We can express this property as a two-dimensional path saying homomorphic encryption of a plain-text concatenated with a path expressing homomorphic multiplication concatenated with homomorphic decryption is the same as the regular addition performed on the plain-text.

The encoding of cryptDB in homotopy type theory involves non-bijective queries. Mapping a non-bijective query into the universe is not possible in the current type-theoretic setting. However, we can map a non-bijective path to singleton types in the universe [2]. Such a mapping holds because any function between singleton types is automatically a bijection.

\subsection{Deterministic Encryption}

Deterministic encryption generates the same cipher-text on multiple encryptions of the same plain-text. In cryptDB, a deterministic encryption scheme is used to perform equality comparisons on encrypted data. The correctness property of deterministic encryption requires $D E T(m 1) \equiv D E T(m 2)$ when $m 1 \equiv m 2$. We can specify this property as a heterogenous path over a path of type $m 1 \equiv m 2$. For example, when tab and det-enc encode the plain-text as 
an implicit argument given by tab : $\{\mathrm{m}\} \rightarrow$ encDB and det-enc $:\{\mathrm{m}\} \rightarrow$ tab $\equiv$ tabDET respectively, we can define the following two-dimensional path.

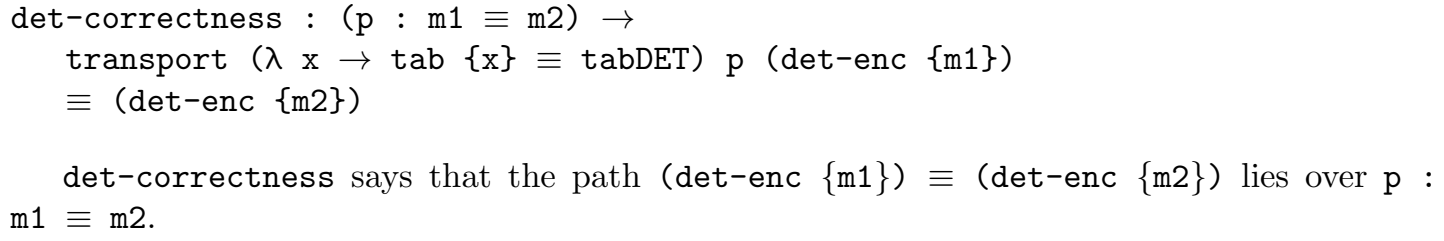

\subsection{Order-Preserving Encryption}

Order-preserving encryption [17] allows inequality comparisons on encrypted data without the need to decrypt them. Order-preserving encryption requires, for plain-texts $x$ and $y$, if $(x<y)$ then $O P E(x)<O P E(y)$. We cannot specify this property in the style of det-correctness because inequality relation does not form paths. However, we can use a different approach to model this restriction in a higher inductive type. For example, consider a function bigE (m1, m2) which returns the biggest of two elements. When there exists a path p' : bigE (m1, m2) $\equiv \operatorname{bigE}(\mathrm{c} 1, \mathrm{c} 2$ ), where $\mathrm{c} 1$ and $\mathrm{c} 2$ are the OPE cipher values of $\mathrm{m} 1$ and $\mathrm{m} 2$ respectively, lying in the space encDB, we can design a two-dimensional path saying ope-encrypt is the same path as p'. This two-dimensional path will hold only when the order-preserving encryption respects the inequality relation between the plain-texts.

The two-dimensional paths discussed above capture different domain-specific laws that should be respected by any concrete implementation of a multi-layered database in the style of cryptDB. By specifying the above paths as constructors of encDB and by mapping encDB to a concrete implementation in the universe similar to OTP in section 3, we can achieve various guarantees on the correctness of the implementation. The mapping of the higher inductive type into the universe alone is enough to guarantee on the correctness of properties specified by the path constructors because of univalence and functoriality. By having a higher inductive type front-end for a cryptographic implementation, we eliminate the need to generate individual proofs for different domain-specific properties. Also in a higher inductive type framework, we have a way to relate proofs of different properties because of the encoding of proofs as paths or higher dimensional paths of a single type.

\section{Implementation}

In cryptDB, functions implementing queries like insert and delete are not bijective and therefore cannot be encoded as paths in a higher inductive type. To address this problem, we used the patch theory [2] approach to encode cryptDB. The higher inductive type representing cryptDB is contractible which allowed us to map the paths representing the non-bijective queries to singleton types in the universe. This mapping is possible since any function between a singleton type is automatically a bijection. We declared the query operations using historytype [2], a higher inductive type that records all the query information. The higher inductive type representing the cryptDB then depends on the history type. We implemented this model in $\mathrm{Agda}^{3}$ by declaring the higher inductive types as rewrite rules [30] using \{-\# REWRITE , ...\#-\} pragma. We automated the code generation for the dependent and non-dependent elimination

\footnotetext{
${ }^{3} \mathrm{~A}$ detailed implementation can be found in the associated github repository. See https://github.com/pavenvivek/LPAR-2018.
} 
rules corresponding to the higher inductive types using an automation tool [33] based on Agda's new support for elaborator reflection [31].

\section{Related Work}

The work discussed in this paper takes the first step towards formal specification of cryptographic protocols based on types. There are other works which support formal specification of cryptographic constructions using different settings for handling cryptographic primitives including shared-key and public-key cryptography, signatures, hash functions, message authentication codes, etc. In this section, we will review few of those works most of which are welldeveloped and have a bigger scope compared to the framework discussed in this paper.

The Foundational Cryptography Framework (FCF) [5] implements a probabilistic programming language embedded inside Coq proof assistant. Unlike Agda, the Coq proof assistant is based on the Calculus of Inductive Construction. However, the recent version of Coq allows the sort Set to be predicative. The probabilistic programming language defined by FCF enables the specification of cryptographic schemes, security definitions, and hard problems. A shallow embedding of the probabilistic language allows FCF to have access to the capabilities of the metalanguage (Coq) including dependent types and higher-order functions.

The work of [22] implemented in CryptoVerif provides a mechanized prover for showing correspondence assertions which are useful to express authentication properties for cryptographic protocols in the computational model. The proof construction follows the sequences of games approach in cryptography. CryptoVerif is based on Process Calculus extended with parametric events to serve in the definition of correspondences. CryptoVerif incorporates efficient automation reducing the proof development effort but lacks interactive proof development features which makes it more specific to only a subset of cryptographic constructions when compared to FCF or EasyCrypt.

ProVerif [21] is a cryptographic protocol verifier for the automated reasoning of security properties based on Dolev-Yao model. It can be used for proving secrecy, authentication, and equivalences between processes differing only by terms. The input protocols to ProVerif are modeled using PiCalculus and internally translated using Horn clauses. The security properties which needs to be proved are translated to derivability queries on these clauses. ProVerif can handle different cryptographic primitives including shared-key and public-key cryptography, hash functions, and Diffie-Hellman key agreements.

CertiCrypt [19], a framework built upon the Coq proof assistant, enables machine-checked construction and verification of cryptographic schemes. The proof development in CertiCrypt is time-consuming, and EasyCrypt [18] was developed to address this limitation by speeding up the construction of proofs using automation based on SMT solvers. Both CertiCrypt and EasyCrypt has a deep embedding of a probabilistic programming language which is used for proof construction. The deep embedding makes them inaccessible to the cozy features of the host language (Coq) such as dependent-types, higher-order functions, modules, etc.

Verypto [14], a framework implemented in Isabelle proof-assistant [22], provides a formal language for the specification and verification of game-based cryptographic security proofs. Verypto includes a probabilistic higher-order functional programming language with recursive types, references, and events to express constructs of a game-based security proof. The language handles stateful higher-order objects such as oracles, arbitrary data types and supports eventbased reasoning patterns. Like CertiCrypt and EasyCrypt, the probabilistic programming language used for proof construction in Verypto follows a deep embedding. 


\section{Limitations and Future Work}

A limitation of homotopy type theory is that the univalence can be added only as an axiom. This limitation weakens the good computational properties of type theory. We would like to develop the technique described in this paper using a particular model of homotopy type theory known as the cubical type theory [27]. In cubical type theory, the univalence computes and is no longer an axiom.

Another limitation is that the mapping of higher inductive type into the universe requires the functions represented by paths to be bijective. We cannot specify all functions as bijections. The functions with simple retractions are not acceptable. Every function should have inverses to be expressed as paths. One way to work around this problem is to encode functions as mappings between singleton types in the universe [2]. Any function mapping between two singleton types is automatically a bijection. So a path representing a non-bijective function in a higher inductive type can be mapped to bijection formed by a function between singleton types in the universe. But this solution can be applied only for contractible types. Future work in this direction would be to characterize mapping of partial bijections to paths using the tools of homotopy theory. Another direction is to develop type theory with non-symmetric paths based on directed type theory [8].

Probabilistic encryption schemes are not bijective. It might not be possible to map them to singleton types in the universe because they compute to different values during each execution with overwhelming probability and does not uniquely identify the contents of a singleton type. Another limitation is the difficulty involved in deriving proofs for bijections. This limitation increases development time and effort. But after application development, we can achieve overwhelming guarantee on the correctness of the application. In the real-world applications, bug fixing has taken much more effort than the original development effort [9][10][11]. So the cost of the increase in development effort can be ignored considering the benefits achieved. It can be very significant especially when implementing cryptographic protocols because a flawed implementation of cryptographic protocol leads to serious security issues resulting in the compromise of the entire application. However, the performance is out of scope for this paper. Agda also has a robust reflection library which can be used to automate the code generation for higher inductive types [33]. Automated code generation can reduce the development effort to some extent. In the future, we would like to encode the security properties of a cryptographic scheme as paths in a higher inductive type and explore how to achieve security guarantees using this setting.

The main purpose of the discussion in this paper is to drive the progress in homotopy type theory research from an application perspective on the programming side. Cryptography is a very significant and vast field, and it would be interesting to see if homotopy type theory can find application in this domain. This paper takes a very first step towards this direction.

\section{Conclusion}

This paper presented a new direction for the formal specification of cryptographic protocols based on types. It gave a real-world application of homotopy type theory in an attempt to solve an important problem in cryptography, namely verifying the correctness of the implementation. It also extended the types in an interesting way by allowing them to act as formal certificates guaranteeing on the correctness properties. Homotopy type theory is still developing and it takes more time and more hard work to get it done. In the meantime, the current features of homotopy type theory such as the higher inductive type and the univalence axiom have been 
put to use by this paper to model an industrial application. Applying homotopy type theory to cryptography is an important topic to explore, and this paper can motivate more research in this direction. In spite of the limited scope of this framework, we still feel this discussion is necessary because the ongoing works [26][27] are promising, and it can motivate more research on the programming side of homotopy type theory.

The limitations of homotopy type theory, namely having univalence only as an axiom and the requirement for functions to have inverses has restricted us to only a subset of cryptographic schemes to be benefitted by the model described in this paper. Nevertheless, there is a lot of work going on to improve type theory to allow for univalence to compute and mapping of nonbijective functions into the universe which can reduce the restrictions and enable us to encode more interesting cryptographic constructions using the higher inductive type model. Also, this paper introduces the tools of homotopy type theory to the cryptographic community and acts as a precursor of more interesting type theoretical settings to follow which can significantly improve the framework described in this paper.

\section{References}

[1] The Univalent Foundations Program, Institute for Advanced Study. Homotopy Type Theory: Univalent Foundations Of Mathematics (2013).

[2] Anguili, C., Morehouse, E., Licata, D., Harper, R.: Homotopical Patch Theory. In: International Conference on Functional Programming (ICFP), Sweden (2014).

[3] Popa, R.A., Redfield, C.M.S, Zeldovich, N., Hari Balakrishnan, H. : CryptDB: Protecting Confidentiality with Encrypted Query Processing. In: Proceedings of the 23rd ACM Symposium on Operating Systems Principles (SOSP), Portugal (2011).

[4] Duan, J., Hurd, J., Li, G., Owens, S., Slind, K., Zhang J.: Functional Correctness Proofs of Encryption Algorithms. In: Proceedings of the 12th international conference on Logic for Programming, Artificial Intelligence, and Reasoning, pp. 519-533. Jamaica (2005).

[5] Petcher, A., Morrisett, G.: The Foundational Cryptography Framework. In: Focardi R., Myers A. (eds) Principles of Security and Trust (POST). Lecture Notes in Computer Science, vol 9036. Springer, Berlin, Heidelberg (2015).

[6] Norell, U.: Towards a practical programming language based on dependent type theory. PhD thesis, Chalmers University of Technology, Sweden (2007).

[7] Licata, D.: Running Circles Around (In) Your Proof Assistant; or, Quotients that Compute. http: //homotopytypetheory.org/2011/04/23/running-circles-around-in-your-proof-assistant (2011).

[8] Licata, D., Harper, R.: 2-dimensional directed type theory. In: Mathematical Foundations of Programming Semantics (MFPS) (2011).

[9] Ben Othmane, L., Chehrazi, G., Bodden, E., Tsalovski, P., Brucker, A.D., Miseldine, P.: Factors Impacting the Effort Required to Fix Security Vulnerabilities. In: Lopez J., Mitchell C. (eds) Information Security (ISC). Springer LNCS. Cham (2015).

[10] Hamill, M., Goseva-Popstojanova, K.: Software faults fixing effort: Analysis and prediction. In: Technical report 20150001332, NASA Goddard Space Flight Center. Greenbelt, MD United States (2014).

[11] Cornell, D.: Remediation statistics: what does fixing application vulnerabilities cost? In: Proceedings of the RSAConference. San Fransisco, CA, USA (2012)

[12] Lazar, D., Chen, H., Wang, X., Zeldovich, N.: Why does cryptographic software fail?: a case study and open problems. In: Proceedings of 5th Asia-Pacific Workshop on Systems (APSys). Beijing, China (2014). 
[13] Durumeric, Z., Kasten, J., Adrian, D., Halderman, J.A., Bailey, M., Li, F., Weaver, N., Amann, J., Beekman, J., Payer, M., Paxson, V.: The Matter of Heartbleed. In: Proceedings of the 2014 Conference on Internet Measurement Conference. Vancouver, BC, Canada (2014).

[14] Berg, M.: Formal Verification of Cryptographic Security Proofs. Ph.D. thesis, Saarland University (2013), http://www.infsec.cs.uni-saarland.de/ berg/publications/thesis-berg.pdf

[15] Kokke, P., Swierstra, W.: Auto in Agda. In: Hinze R., Voigtländer J. (eds) Mathematics of Program Construction (MPC). Springer LNCS, vol 9129. Cham (2015).

[16] Paillier, P.: Public-key cryptosystems based on composite degree residuosity classes. In: Proceedings of the 18th Annual International Conference on the Theory and Applications of Cryptographic Techniques (EUROCRYPT). Prague, Czech Republic (1999).

[17] Agrawal, R., Kiernan, J., Srikant, R., and Xu, Y.: Order preserving encryption for numeric data. In Proceedings of the 2004 ACM SIGMOD International Conference on Management of Data. Paris, France (2004).

[18] Gilles, B., Grégoire, B., Heraud, S., and Béguelin, S.Z.: Computer-aided security proofs for the working cryptographer. In: Advances in Cryptology - CRYPTO 2011. Lecture Notes in Computer Science, vol. 6841, pp. 71-90 (2011).

[19] Gilles, B., Grégoire, B., and Béguelin, S.Z.: Formal certification of code-based cryptographic proofs. In: 36th ACM SIGPLAN-SIGACT Symposium on Principles of Programming Languages, POPL 2009. pp. 90-101 (2009).

[20] Blanchet, B.: Computationally sound mechanized proofs of correspondence assertions. In: 20th IEEE Computer Security Foundations Symposium (CSF'07), pp.97-111. Venice, Italy (2007).

[21] Blanchet, B.: Modeling and Verifying Security Protocols with the Applied Pi Calculus and ProVerif. In: Foundations and Trends in Privacy and Security. vol. 1, num. 1-2, pp.1-135 (2016).

[22] Nipkow, T., Paulson L. C., and Wenzel, M.: Isabelle/HOL - A Proof Assistant for Higher-Order Logic. Lecture Notes in Computer Science, vol. 2283, Springer (2002).

[23] Russell, B.: The Principles of Mathematics: WW Norton \& Company (1996).

[24] Martin-Löf, P.: "An Intuitionistic Theory of Types: Predicative Part". Studies in Logic and the Foundations of Mathematics; 80 (1975), 73-118.

[25] Martin-Löf, P.: "Constructive Mathematics and Computer Programming”. Studies in Logic and the Foundations of Mathematics; 104 (1982), 153-175.

[26] Angiuli, C., Harper, R., and Wilson, T.: Computational Higher-dimensional Type Theory. Proceedings of the 44th ACM SIGPLAN Symposium on Principles of Programming Languages (POPL'17), Paris, France (2017).

[27] Cohen, C., Coquand, T., Huber, S., and Mörtberg, A.: Cubical Type Theory: a constructive interpretation of the univalence axiom. 21st International Conference on Types for Proofs and Programs (2015).

[28] Altenkirch, T.: Containers in homotopy type theory. Talk at Mathematical Structures of Computation, Lyon (2014).

[29] Abbott M., Altenkirch, T. and Ghani, N.: Containers: constructing strictly positive types. Theoretic Computer Science (2005).

[30] Cockx, J. and Abel, A.: Sprinkles of Extensionality for Your Vanilla Type Theory. 22nd International Conference on Types for Proofs and Programs (TYPES 2016).

[31] Christiansen, D. and Brady, E.: Elaborator Reflection: Extending Idris in Idris. In: Proceedings of the 21st ACM SIGPLAN International Conference on Functional Programming (ICFP '16). Nara, Japan (2016).

[32] Roundy, D.: Darcs: Distributed version management in haskell. ACM SIGPLAN Workshop on Haskell (2005).

[33] Vivekanandan, P.: Code Generation for Higher inductive Types. The 26th International Workshop on Functional and Logic Programming (WFLP'18). Frankfurt, Germany (2018). 